\title{
Erratum: One-Way Quantum Repeater Based on Near-Deterministic Photon-Emitter Interfaces [Phys. Rev. X 10, 021071 (2020)]
}

\author{
Johannes Borregaard®, Hannes Pichler, Tim Schröder, Mikhail D. Lukin, Peter Lodahl, and Anders S. Sørensen
}

(Received 4 February 2021; published 14 June 2021)

DOI: 10.1103/PhysRevX.11.029903

After the publication of the article, we unfortunately discovered an error in the displayed rate for a generic two-way repeater, which we compared our results to in Figs. 6(c) and 6(d). This error does not affect the one-way repeater, which is the main focus of our work.

As described in the Supplemental Material of the article, we performed an optimization of the secret key rate of a generic two-way repeater under the constraint that the total number of spin qubits used in the two-way repeater was not larger than the total number of spin qubits in the one-way repeater for the same distance. Specifically, we estimated the secret key rate $r$ as

$$
r=\frac{1}{Z_{l}\left(p_{\text {ent }}\right)} \times \frac{(l+1) c}{L},
$$

where $Z_{l}(p)=\sum_{k=1}^{l+1}\left(\begin{array}{c}l+1 \\ k\end{array}\right)\left[(-1)^{k+1} / 1-(1-p)^{k}\right]$ accounts for the number of tries to generate entanglement in $l+1$ pairs, where each pair succeeds with probability $p$ [1]. The success probability of entanglement generation in a link was estimated as

$$
p_{\mathrm{ent}} \approx 1-\left(1-\frac{1}{2} \eta_{d}^{2} e^{-\left(L /(l+1) L_{\mathrm{att}}\right.}\right)^{\lceil(3(m+1)) /(2(l+1))]},
$$

which is the probability that at least one entangled pair is generated using $\lceil(3(m+1)) /(2(l+1))\rceil$ parallel attempts. Here, we used the ceiling function \lceil\rceil to get an upper bound. We then optimized the secret key rate as a function of the number of equally distributed repeater stations $l$.

Unfortunately, an unintentional upper bound of $l \leq 31$ was enforced in the optimization quoted in the article. The rates for the two-rate repeater in Figs. 6(c) and 6(d) of the article are therefore subject to this constraint.

In Fig. 1, we reproduce Figs. 6(c) and 6(d) from the article but include the results of a new optimization of the two-way repeater without the constraint of $l \leq 31$. However, we have reenforced that the spacing between repeater stations is greater than or equal to $1 \mathrm{~km}$, as for the one-way repeater.

The optimal number of repeater stations corresponding to the optimization is shown in Fig. 2. Because we assume perfect entanglement generation and swapping, it becomes favorable to have many repeater nodes resulting in even more nodes than for the one-way repeater (see Fig. S1 in Supplemental Material of the paper for numbers for the one-way repeater).

The use of the ceiling function in Eq. (2) to get an upper bound results in roughly a factor of 2 higher total number of spin qubits for the optimized two-way repeater than for the one-way repeater. To ensure that the total number of qubits used is not larger than the total number of spin qubits in the one-way repeater for the same distance, we can change Eq. (2) to

$$
p_{\text {ent }} \approx 1-\left(1-\frac{1}{2} \eta_{d}^{2} e^{-\left(L /(l+1) L_{\text {att }}\right)}\right)^{\text {quotient }[3(m+1), 2(l+1)]},
$$

where quotient $[a, b]=k$, such that $a=k \cdot b+r$, with $k$ being an integer and $0 \leq r \leq b$. The result of an optimization of the two-way repeater using Eq. (3) instead of Eq. (2) is also shown in Figs. 1 and 2. Notably, this reduces the rates by about a

Published by the American Physical Society under the terms of the Creative Commons Attribution 4.0 International license. Further distribution of this work must maintain attribution to the author(s) and the published articles title, journal citation, and DOI. 

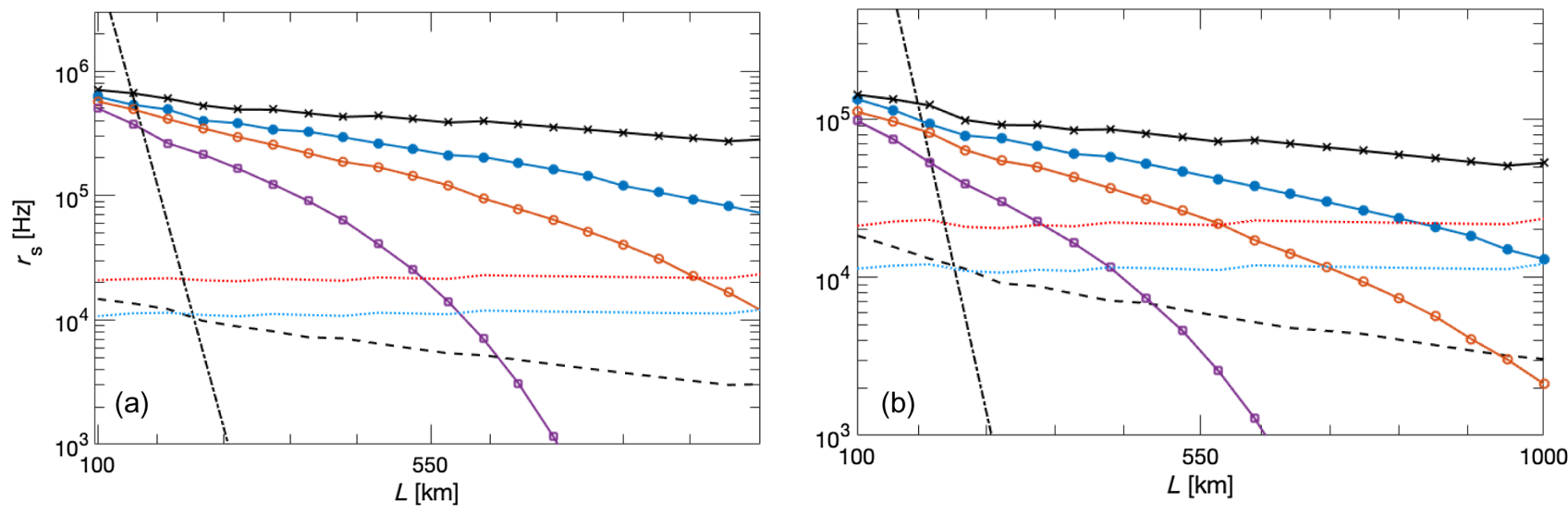

FIG. 1. Reproduction of Figs. 6(c) and 6(d) from the main article, with corrected simulations of the two-way repeater added. Panel (a) corresponds to Fig. 6(c) and panel (b) to Fig. 6(d) of the article. The black dashed line is the published erroneous rate of the generic twoway repeater, while the red dashed lines show the result of the corrected rate optimization. The blue dashed lines show the result of an optimization, where the total number of qubits is kept strictly the same or lower than the total number of spin qubits for the one-way repeater for the same distance. The specifics of the other curves correspond to those of Figs. 6(c) and 6(d) in the article. The secret bit rate of the tree repeater, assuming a photon emission time of $\tau_{\mathrm{ph}}=1 \mathrm{~ns}$ and gate time of $\tau_{\mathrm{CZ}}=10 \mathrm{~ns}$, is shown in panel (a), while for a gate time of $\tau_{\mathrm{CZ}}=100 \mathrm{~ns}$, it is shown in panel (b). The markers correspond to the following: $\times: \epsilon_{r}=0.1 \%$ o, $\bullet: \epsilon_{r}=0.3 \%$, ० : $\epsilon_{r}=0.5 \%$, and $\square: \epsilon_{r}=0.1 \%$. Here, $\epsilon_{r}$ is the error probability of the re-encoding operation at the repeater stations. In comparison, we have assumed the generic two-way repeater to be error-free. We have restricted the minimization to trees with $n \leq 300$ photons and repeater station spacings greater than or equal to $1 \mathrm{~km}$. We have assumed a detection efficiency of $\eta_{d}=95 \%$. For comparison, the secret bit rate of direct transmission (dot-dashed line) is shown, assuming a 1-GHz single-photon source.
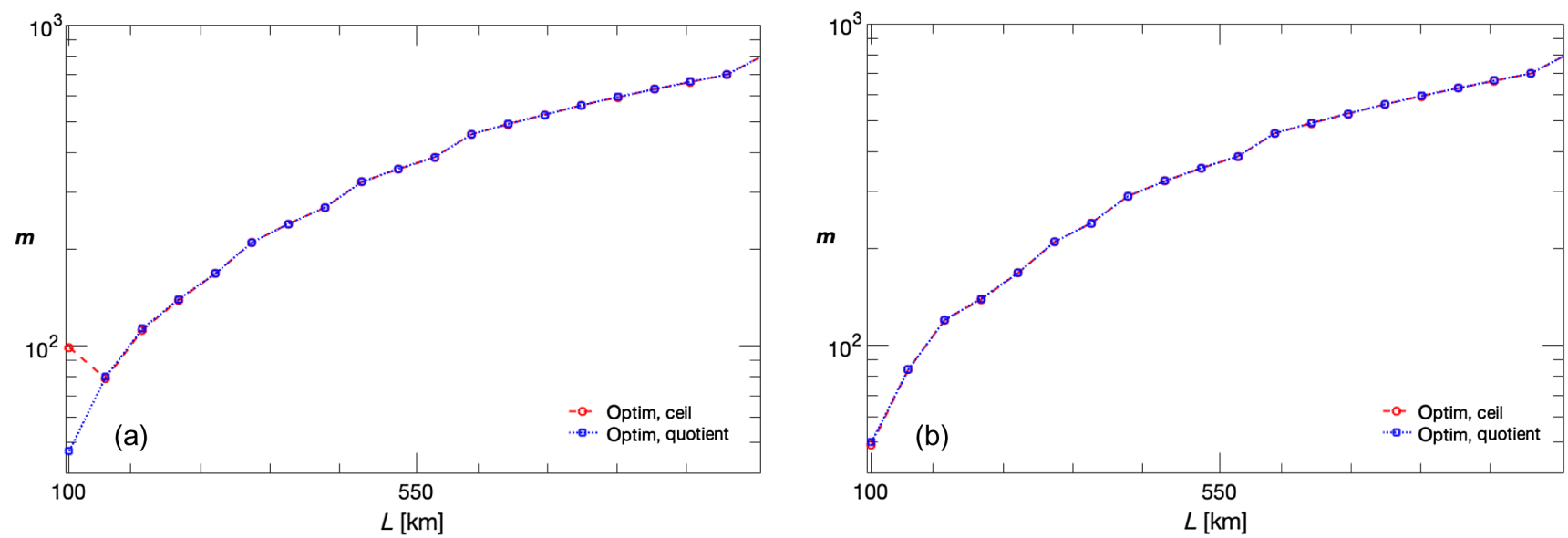

FIG. 2. Optimal number of repeater stations $(\mathrm{m})$ corresponding to the new optimizations of the generic two-way repeater shown in Fig. 1. Panels (a) and (b) correspond to Figs. 1(a) and E1(b). In the figure legends, "optim, ceil" ("optim, quotient") refers to an optimization using the ceiling (quotient) function. We note that the optimal number of repeater stations is very similar for both optimizations, resulting in almost overlapping curves. For the ceiling function, the number of spin qubits per repeater station is found to be 4 for all distances in panels (a) and (b) except $d=100$ in (a). For the quotient function, the optimal number of spin qubits per repeater station is 2 for all distances in both (a) and (b).

factor of 2, while the optimal number of repeater stations stays roughly the same. We also note that the total number of spin qubits for the optimal two-way repeater is found to be either equal to or one less than the total number of spin qubits for the one-way repeater for the same distance in these optimizations.

As shown in Fig. 1, the new optimization improves the two-way repeater by up to an order of magnitude for the considered parameters. For the lowest error rates considered for the one-way repeater, it still maintains a substantial advantage over the ideal two-way repeater. As opposed to the previous optimization, the two-way repeater now involves a much larger number of repeater stations. Thus, the assumption of neglecting errors in the two-way repeater is questionable 
for the error rates considered here. Had we included errors of a similar size as for the one-way repeater in the two-way repeater, it would decrease its rate, making the difference between the two approaches even larger. The main conclusion that the one-way repeater outperforms the two-way repeater for sufficiently low error rates is thus still valid.

[1] Nadja K. Bernardes, Ludmiła Praxmeyer, and Peter van Loock, Rate Analysis for a Hybrid Quantum Repeater, Phys. Rev. A 83, 012323 (2011). 